\title{
Parameter identification for lined tunnels in a viscoplastic medium
}

\author{
B. Lecampion, A. Constantinescu and D. Nguyen Minh*,† \\ Laboratoire de Mécanique des Solides, CNRS URA 7649, Ecole Polytechnique, 91128 Palaiseau Cedex, France
}

\begin{abstract}
This paper is dedicated to the identification of constitutive parameters of elasto-viscoplastic constitutive law from measurements performed on deep underground cavities (typically tunnels). This inverse problem is solved by the minimization of a cost functional of least-squares type. The exact gradient is computed by the direct differentiation method and the descent is done using the Levenberg-Marquardt algorithm. The method is presented for lined or unlined structures and is applied for an elastoviscoplastic constitutive law of the Perzyna class. Several identification problems are presented in one and two dimensions for different tunnel geometries. The used measurements have been obtained by a preliminary numerical simulation and perturbed with a white noise. The identified responses match the measurements. We also discuss the usage of the sensitivity analysis of the system, provided by the direct differentiation method, for the optimization of in situ monitoring. The sensitivity distribution in space and time assess the location of the measurements points as well as the time of observation needed for reliable identification.
\end{abstract}

KEY WORDS: identification; viscoplasticity; underground structures; tunnel; sensitivity analysis; inverse problem

\section{INTRODUCTION}

It is well known that underground structures located in salt, hard marls, etc. present important time-dependent deformations even dozens of years after the excavation. Several examples of case histories can be found in the literature: large delayed displacement have been reported in motorway tunnels [1], or long-term subsidence has been observed over salt caverns [2]. A series of industrial problems involving deep cavities, like the storage of radioactive waste, depend on the characterization of such effects in order to assess the safety of the overall project.

These time-dependent observations are generally explained by several phenomena: hydromechanical couplings [3] and/or rate-dependent mechanical behaviour [4,5]. Predictive models are classically proposed from a series of laboratory tests. Unfortunately, in many cases there is

\footnotetext{
*Correspondence to: D. Nguyen Minh, Laboratoire de Mécanique des Solides, CNRS URA 7649, Ecole Polytechnique, 91128 Palaiseau Cedex, France.

†E-mail: duc@1ms.polytectnique.fr

Contract/grant sponsor: French Nuclear Agency for Radioactive Waste Management (ANDRA)
} 
still a discrepancy between predictions and measurements when one passes from the laboratory to the field.

These discrepancies can be justified by a series of factors: scale effects, uncertainties on the constitutive model itself, on the identified parameters of the chosen constitutive law, etc. It is obvious that it is preferable to correct the constitutive parameters using the first measurements already available before any long-term extrapolation of the model.

The problem addressed in this paper is the identification (or correction) of the constitutive parameters from field measurements. The work presented here is a further attempt to propose a systematic method to reduce the gap between field observations and computations performed with the parameters identified from results of laboratory tests.

The problem of parameter identification is an inverse problem [6,7], also known as backanalysis. In general, the problem is defined as the minimization of a cost functional measuring the distance between measurements and predictions. A large review of back-analysis methods in geotechnical engineering is presented in Reference [8].

In this work, we shall only refer to gradient-based minimization; however, it is important to notice that a large class of evolutionary methods (genetic algorithms, neural networks, etc.) have been proposed recently in the literature. Our choice of gradient based minimization is justified on the one hand by the computational burden of the evolutionary methods and on the other hand by the form of the cost functional in the explored parameter space (see Figure 10).

Most of the results in geotechnics have been obtained in the case of linear material behaviour [9-11]. This is mainly due to the computational burden and the intrinsic complexity of the inverse procedure for the non-linear behaviour. A small number of papers deal with an elastoplastic constitutive law. They generally use an optimization procedure without gradient [12] due to the difficulties of the differentiation of the elastoplastic inequalities.

When the gradients are provided by the finite difference method, we dispose only of an approximate value. Moreover, it is well known that this method presents two main drawbacks, one comes from the difficult choice of the step size in computing the difference and the other one stems from the large number of non-linear problems which have to be solved.

Recently, a series of papers (for a general introduction see Reference [13]) have shown that one can solve the identification problems using an exact gradient computation as compared with finite differences. The exact gradient can be obtained by solving a series of additional problems using one of the two following methods:

- In the adjoint state method one constructs a single additional problem, the so-called adjoint problem which is linear, i.e. viscoelastic, and has to be integrated backwards in time. The solution of the direct problem and of the adjoint problem determines the exact value of the gradient, independent of the number of parameters in the minimization process.

- An example of a solution for the case of an elastoviscoplastic law can be found in Reference [14].

- In the direct differentiation method one constructs an additional problem for each parameter involved in the minimization process, which is linear, i.e. viscoelastic, and has to be integrated forward in time. This can be done at each load increment after the integration of the direct problem. The local sensitivities of all fields with respect to all parameters are obtained and can be used for the optimization.

This method has been previously used in a series of papers by Vidal et al. [15,16], Tsay and Arora [17], Galarza et al. [18] in order to solve optimization problems. 
In the sequel, we shall present a solution to the identification problem of material parameters in the case of underground structures using the direct differentiation method. An important step is the coupling of the sensitivity analysis with the numerical modelling of the tunneling process including the set up of the lining. As a first approach, we shall neglect the hydromechanical coupling and focus just on the mechanical behaviour of the rock mass. We refer to Bérest [4] and Cristescu [5] for a complete review of the viscoplasticity of rocks.

In the first part, direct differentiation method is derived for elastoviscoplastic constitutive law of the Perzyna type [19] following the steps of Vidal et al. [15]. In the second part, the method is tested on a series of examples for a Norton-Hoff material law using simulated measurements.

The results obtained using a Levenberg-Marquardt minimization show that the method is robust and efficient. However, certain questions about the uniqueness and stability of the inverse problem also arise. We shortly discuss the usage of the sensitivity analysis of the system for the optimization of in situ monitoring. The sensitivity distribution of the mechanical fields in space and time assess the location of the measurements points as well as the time of observation needed for reliable identification.

\section{THE LINED TUNNEL AND THE ASSOCIATED INVERSE PROBLEMS}

Let us consider the following time interval $T=\left[0, t_{f}\right]$ during which the tunnel will be excavated, supported and monitored. The lining will be installed at $t=t_{\ell}$.

The domain of interest $\Omega$ evolves during this period in the following way:

- initially, for $t \in[-\infty, 0[, \Omega$ is the non-excavated domain;

- then, for $t \in\left[0, t_{l}\right]$ the domain $\Omega=\Omega_{X}$ represents the rock mass with the excavated tunnel before the installation of the lining;

- finally, for $t>t_{l}$, the domain $\Omega=\Omega_{X} \cup \Omega_{\ell}$ is the excavated rock mass together with the lining of the tunnel.

The border $\Gamma=\partial \Omega$ of the complete domain $\Omega$ is divided into two sets $\Gamma_{t}$ and $\Gamma_{u}$ on which tractions and displacements are, respectively, prescribed. The interface between the lining and the rock mass will be denoted $\Gamma_{X \ell}$.

Let $\mathbf{u}, \boldsymbol{\varepsilon}$ and $\boldsymbol{\sigma}$ stand for the displacement, the small strain operator and the Cauchy stress tensor, respectively.

\subsection{The direct problem $\mathscr{P}$}

Before excavation, at time $t=0$, we shall assume that the rock mass is in its natural state given by the initial statically admissible stress state:

$$
\boldsymbol{\sigma}=\boldsymbol{\sigma}_{0}
$$

and that all the other mechanical fields are equal to zero. The evolution of the rock mass is described by the following system of equations, written in rate form as

- Balance equation on $\Omega \times T$ and imposed tractions on the boundary on $\Gamma_{t} \times T$ :

$$
\begin{gathered}
\operatorname{div} \dot{\boldsymbol{\sigma}}+\dot{\mathbf{f}}=0 \\
\dot{\boldsymbol{\sigma}} \cdot \mathbf{n}=\dot{\mathbf{t}}^{\mathrm{d}}
\end{gathered}
$$


- Small strain equations on $\Omega \times T$ and displacement boundary conditions on $\Gamma_{\mathbf{u}} \times T$ :

$$
\begin{aligned}
& \varepsilon(\dot{\mathbf{u}})=\frac{1}{2}\left(\nabla \dot{\mathbf{u}}+\nabla^{\mathrm{T}} \dot{\mathbf{u}}\right) \\
& \dot{\mathbf{u}}=\dot{\mathbf{u}}^{\mathrm{d}}
\end{aligned}
$$

- Elastoviscoplastic constitutive law of Perzyna class [19] for the rock mass on $\Omega_{X} \times T$ :

$$
\begin{aligned}
& \dot{\boldsymbol{\sigma}}=\mathbf{C}:\left(\dot{\boldsymbol{\varepsilon}}-\dot{\boldsymbol{\varepsilon}}^{\mathrm{vp}}\right) \\
& \dot{\boldsymbol{\varepsilon}}^{\mathrm{vp}}=\frac{\partial \phi(\boldsymbol{\sigma})}{\partial \boldsymbol{\sigma}}
\end{aligned}
$$

where $\phi$ is a pseudo-potential functional of the stress tensor [19].The expression of $\phi$ for the Norton-Hoff law is given by

$$
\phi(\boldsymbol{\sigma})=\frac{K}{N+1}\left\langle\frac{\sigma_{\mathrm{eq}}-\sigma_{\mathrm{Y}}}{K}\right\rangle^{N+1}
$$

where $\sigma_{\mathrm{eq}}$ is the Von Mises equivalent stress $\left[M L^{-1} T^{-2}\right] ; \sigma_{\mathrm{Y}}$ the yield limit $\left[M L^{-1} T^{-2}\right] ; N$ the viscoplastic exponent [.]; and $K$ the viscosity coefficient $\left[M L^{-1} T^{-2+1 / N}\right]$.

- Elastic behaviour for the lining on $\left.\left.\Omega_{\ell} \times\right] t_{l}, t_{f}\right]$ :

$$
\dot{\boldsymbol{\sigma}}=C_{l}: \dot{\boldsymbol{\varepsilon}}
$$

The constitutive law is fairly simple, but includes several interesting features: a cohesion and a highly non-linear viscosity. A series of classical characteristics such as plastic dilatancy or hardening/softening effects are not included. However, this material behaviour reproduces the shape of the delayed convergence of tunnels excavated in viscoplastic rocks.

Moreover in rock salt mechanics, it is largely accepted that the material behaves like a viscous fluid. The non-Newtonian Norton or Lemaitre-Menzel-Schreiner constitutive laws are frequently used in applications [20]. Other viscous rocks such as shales or marls cannot be considered as viscous fluids and a long-term resistance must be accounted for. In this case, the larger class of viscoplastic constitutive laws of Perzyna type has been proposed by several authors $[1,21]$. One of these, the Norton-Hoff law used here for illustration permits a direct extension to include strain hardening (or softening) as well as internal friction.

The numerical integration of the initial boundary value problem is classically done by finite elements in space and finite difference in time. An extensive presentation of these techniques is given in Reference [22].

At this point, let us recall the weak formulation of the preceding problem which will be used in the sequel for the description of the direct differentiation method. On the discretized time interval $\left[t_{n}, t_{n+1}\right]$, the weak formulation at time $t=t_{n+1}$ can be written using a forward Euler (implicit) scheme for the time integration and a radial return mapping for the local plastic integration [22]:

Find $\mathbf{u}_{n+1} \in \mathscr{U}, \boldsymbol{\varepsilon}_{n+1}^{\mathrm{vp}}$ and $\boldsymbol{\sigma}_{n+1}$ satisfying :

$$
\begin{aligned}
& \int_{\Omega} \boldsymbol{\varepsilon}(\mathbf{v}): \boldsymbol{\sigma}_{n+1} \mathrm{~d} \Omega-\int_{\Omega} \mathbf{v f}_{n+1} \mathrm{~d} \Omega-\int_{\Gamma_{t}} \mathbf{v t}_{n+1}^{d} \mathrm{~d} \Gamma=0 \quad \forall \boldsymbol{v} \in \mathscr{V} \\
& \boldsymbol{\sigma}_{n+1}=\mathbf{C}:\left(\boldsymbol{\varepsilon}\left(\mathbf{u}_{n+1}\right)-\boldsymbol{\varepsilon}_{n+1}^{\mathrm{vp}}\right)+\boldsymbol{\sigma}_{0} \\
& \boldsymbol{\varepsilon}_{n+1}^{\mathrm{vp}}=\boldsymbol{\varepsilon}_{n}^{\mathrm{vp}}+\Delta t_{n+1} \frac{\partial \phi\left(\boldsymbol{\sigma}_{n+1}\right)}{\partial \boldsymbol{\sigma}}
\end{aligned}
$$


where all fields $X\left(t_{n+1}\right)$ at time $t=t_{n+1}$ are noted $X_{n+1}$ and $\Delta t_{n+1}=t_{n+1}-t_{n}$.

$\mathscr{U}$ and $\mathscr{V}$ are the classical functional space :

$$
\begin{gathered}
\mathscr{U}=\left\{\mathbf{u} \in \mathbf{H}^{1}(\Omega) \mid \mathbf{u}_{n+1}=\mathbf{u}^{d}\left(t_{n+1}\right) \text { on } \Gamma_{u}\right\} \\
\mathscr{V}=\left\{\mathbf{v} \in \mathbf{H}^{1}(\Omega) \mid \mathbf{v}=0 \text { on } \Gamma_{u}\right\}
\end{gathered}
$$

We recall that $\boldsymbol{\sigma}_{\mathbf{0}}$ denotes the in situ stresses field.

Let us now discuss the computational details imposed by the excavation of the tunnel and the installation of the lining. The finite element computations will be performed on the domain $\Omega_{X} \cup \Omega_{\ell}$ for all time steps. Before the effective installation of the lining, the mechanical influence of $\Omega_{\ell}$ has to be neglected and therefore Young's moduli of the lining has been chosen such that $E_{l} \ll E_{m}$.

The different steps can now be described in the following way:

- For $t \in[-\infty, 0[$, the non-excavated domain is at equilibrium with an initial stress state. The 'finite element' boundary condition should be: $\left.\boldsymbol{\sigma} \cdot \mathbf{n}\right|_{\Gamma_{\ell}}=\boldsymbol{\sigma}_{\mathbf{0}} \cdot \mathbf{n}$. Due to the negligible stiffness in $\Omega_{\ell}$ this is practically equivalent with the real condition: $\left.\boldsymbol{\sigma} \cdot \mathbf{n}\right|_{\Gamma_{X \ell}}=\boldsymbol{\sigma}_{0} \cdot \mathbf{n}$.

- For $t \in\left[0, t_{\ell}[\right.$ : The tunnel is already excavated but the lining is not active. The boundary $\Gamma_{X \ell}$ of the rock mass is free of traction. Using the same argument as before, we impose: $\left.\boldsymbol{\sigma} \cdot \mathbf{n}\right|_{\Gamma_{X \ell}}=\mathbf{0}$. Due to the negligible stiffness in $\Omega_{\ell}$ this is practically equivalent with the real condition: $\left.\boldsymbol{\sigma} \cdot \mathbf{n}\right|_{\Gamma_{X \ell}}=\mathbf{0}$.

- For $t \in\left[t_{\ell}, t_{f}\right]$, the elastic lining is set. The boundary condition is now: $\left.\boldsymbol{\sigma} \cdot \mathbf{n}\right|_{\Gamma_{\ell}}=\mathbf{0}$. However, at this point the stiffness of the lining is not negligible anymore and therefore the traction between lining and rock mass expressed by $\left.\boldsymbol{\sigma} \cdot \mathbf{n}\right|_{\Gamma_{X \ell}}$ is no more zero.

Here, we supposed that an instantaneous de-confinement is applied at the tunnel wall to simulate the excavation process. This is justified by the fact that excavation period, typically some weeks, is negligible with respect to the period of interest, typically 10-50 years. An extended discussion supporting these assumptions from the geomechanical point of view can be found in Reference [23].

Taking into account a complicated history of de-confinement does not change fundamentally our approach, but for the sake of simplicity this problem will not be addressed thereafter.

\subsection{The inverse problem $\mathscr{P}^{-1}$}

In the inverse problem, we shall consider that the constitutive parameters are unknown. This drawback will be supplemented with a series of measurements, for example:

- convergence of the tunnel wall;

- displacement measurements, by extensometers, within the rock mass and

- pressure cells, measuring the contact forces between the lining and the rock mass.

A general formulation of this identification problem is

Find $\mathbf{c}=\left\{E, N, \sigma_{Y}, K\right\}$ from displacements and forces measurements performed during the time interval $T$. 
From a mathematical point of view, this problem can be recasted as the minimization of a cost functional measuring the gap between measurements and the direct estimations.

In order to solve this problem two issues have to be addressed:

- Choice of the cost functional.

- Choice of the minimization algorithm.

In our case, we have chosen to minimize the classical weighted least-squares functional:

$$
\begin{aligned}
\mathscr{J}(\mathbf{c})= & \frac{1}{2} \sum_{i=1, k} \sum_{j=1, p}\left(\frac{\mathbf{u}\left(\mathbf{c}, \mathbf{t}_{i}, \mathbf{x}_{j}\right)-\mathbf{u}_{\mathrm{mes}}\left(\mathbf{t}_{i}, \mathbf{x}_{j}\right)}{w_{u_{i j}}}\right)^{2} \\
& +\frac{1}{2} \sum_{i=1, k} \sum_{j=1, p}\left(\frac{\mathbf{t}\left(\mathbf{c}, t_{i}, \mathbf{x}_{j}\right)-\mathbf{t}_{\mathrm{mes}}\left(t_{i}, \mathbf{x}_{j}\right)}{w_{t_{i j}}}\right)^{2}
\end{aligned}
$$

where $\mathbf{u}$ and $\mathbf{t}$ are displacements and tractions computed by direct computations, $\mathbf{u}_{\text {mes }}$ and $\mathbf{t}_{\text {mes }}$ are measured quantities, $t_{i}, \mathbf{x}_{j}$ are, respectively, the time instants and the location of the measurements, $w_{t}$ and $w_{u}$ are weights.

The gradient of the preceding cost functional can be written as

$$
\begin{aligned}
\frac{\partial \mathscr{J}}{\partial c_{l}}= & \sum_{i=1, k} \sum_{j=1, p} \frac{\mathbf{u}\left(\mathbf{c}, \mathbf{t}_{i}, \mathbf{x}_{j}\right)-\mathbf{u}_{\mathrm{mes}}\left(t_{i}, \mathbf{x}_{j}\right)}{w_{u_{i j}}^{2}} \frac{\partial \mathbf{u}}{\partial c_{l}}\left(\mathbf{c}, \mathbf{t}_{i}, \mathbf{x}_{j}\right) \\
& +\sum_{i=1, k} \sum_{j=1, p} \frac{\mathbf{t}\left(\mathbf{c}, \mathbf{t}_{i}, \mathbf{x}_{j}\right)-\mathbf{t}_{\mathrm{mes}}\left(\mathbf{t}_{i}, \mathbf{x}_{j}\right)}{w_{t_{i j}}^{2}} \frac{\partial \mathbf{t}}{\partial c_{l}}\left(\mathbf{c}, \mathbf{t}_{i}, \mathbf{x}_{j}\right)
\end{aligned}
$$

In the sequel, we shall use the direct differentiation method to compute the gradient. More precisely, this method allows to compute exactly not only $\partial \mathscr{J} / \partial \mathbf{c}$ as in the adjoint state method but also the values of the sensitivities of all mechanical fields $\partial \mathbf{u} / \partial c_{l}, \partial \boldsymbol{\sigma} / \partial c_{l}, \ldots$ in each point and at each instant. This method requires the solution of $n$ additional viscoelastic problems, where $n$ is the number of parameters. This method will be described in the next section.

\section{SENSITIVITY ANALYSIS}

We will describe the Direct differentiation method for a general set of parameter $\mathbf{c}=$ $\left(c_{1}, \ldots, c_{i} \ldots\right)$ and consider the particular case of constitutive parameters at the end. The direct differentiation of the classical initial boundary problem in elasto-viscoplasticity will rise a somehow similar system of partial differential equations, where the constitutive equation is always similar to a viscoelastic law. The unknown functions of these problems are $\delta_{c_{i}} \mathbf{u}$ and $\delta_{c_{i}} \boldsymbol{\sigma}$. They represent, respectively, the gradients $\partial \mathbf{u} / \partial c_{i}$ and $\partial \boldsymbol{\sigma} / \partial c_{i}$.

The differentiation of the equilibrium and compatibility equations are straightforward since the divergence and gradient are linear operators. The boundary and initial conditions are also easily derived. In particular, if the boundary conditions do not depend on the constitutive parameters $c_{i}$, the sensitivity problem has homogeneous boundary conditions. 


\subsection{Differentiation of the discretized constitutive law}

The pseudo-potential is a function of the parameters $c_{i}$ and the stress field also depends on $c_{i}$. Therefore, we have $\phi\left(\sigma\left(c_{i}\right), c_{i}\right)$.

The differentiation of Equations (5) and (4) thus gives:

$$
\begin{gathered}
\delta_{c_{i}} \boldsymbol{\sigma}_{n+1}=\frac{\partial \mathbf{C}}{\partial c_{i}}:\left(\boldsymbol{\varepsilon}\left(\mathbf{u}_{n+1}\right)-\boldsymbol{\varepsilon}_{n+1}^{\mathrm{vp}}\right) \\
+\mathbf{C}:\left(\boldsymbol{\varepsilon}\left(\delta_{c_{i}} \mathbf{u}_{n+1}\right)-\delta_{c_{i}} \boldsymbol{\varepsilon}_{n+1}^{\mathrm{vp}}\right)+\frac{\partial \boldsymbol{\sigma}_{0}}{\partial c_{i}} \\
\delta_{c_{i}} \boldsymbol{\varepsilon}_{n+1}^{\mathrm{vp}}=\delta_{c_{i}} \boldsymbol{\varepsilon}_{n}^{\mathrm{vp}}+\Delta t_{n+1} \frac{\partial^{2} \phi\left(\boldsymbol{\sigma}\left(c_{i}\right), c_{i}\right)}{\partial \boldsymbol{\sigma} \partial c_{i}}+\Delta t_{n+1} \frac{\partial^{2} \phi\left(\boldsymbol{\sigma}_{n+1}\right)}{\partial \boldsymbol{\sigma}^{2}} \delta_{c_{i}} \boldsymbol{\sigma}_{n+1}
\end{gathered}
$$

Combining the last equations, we can derive the following constitutive law for the sensitivity fields (see details in the Appendix):

$$
\begin{aligned}
\delta_{c_{i}} \boldsymbol{\sigma}_{n+1}= & \Xi_{n+1}: \boldsymbol{\varepsilon}\left(\delta_{c_{i}} \mathbf{u}_{n+1}\right) \\
& +\Xi_{n+1}:\left[\mathbf{C}^{-1}: \frac{\partial \mathbf{C}}{\partial c_{i}}:\left(\boldsymbol{\varepsilon}\left(\mathbf{u}_{n+1}\right)-\boldsymbol{\varepsilon}_{n+1}^{\mathrm{vp}}\right)\right] \\
& +\Xi_{n+1}:\left[\mathbf{C}^{-1}: \frac{\partial \boldsymbol{\sigma}_{0}}{\partial c_{i}}-\delta_{c_{i}} \boldsymbol{\varepsilon}_{n}^{\mathrm{vp}}-\frac{\partial^{2} \phi\left(\boldsymbol{\sigma}_{n+1}\left(c_{i}\right), c_{i}\right)}{\partial \boldsymbol{\sigma} \partial c_{i}} \Delta t_{n+1}\right]
\end{aligned}
$$

where $\boldsymbol{\Xi}_{n+1}$ is the consistent tangent operator [22, 15] defined by

$$
\boldsymbol{\Xi}_{n+1}=\left(\mathbf{C}^{-1}+\Delta t_{n+1} \frac{\partial^{2} \phi\left(\boldsymbol{\sigma}_{n+1}\right)}{\partial \boldsymbol{\sigma}^{2}}\right)^{-1}
$$

This constitutive relation is of the visco-elastic type, the response of the stress sensitivity at time $t_{n+1}$ depends linearly on the displacement sensitivity at time $t_{n+1}$ and known sensitivities at $t_{n}$.

\subsection{Weak form of the sensitivity problem $\mathscr{P}^{s}$}

Following a similar method as in the direct problem, the initial boundary value problem on the sensitivity can be written in the following weak form:

$$
\begin{gathered}
\text { Find } \delta_{c_{i}} \mathbf{u}_{n+1} \in \mathscr{W} \text { and } \delta_{c_{i}} \boldsymbol{\sigma}_{n+1} \text { satisfying : } \\
\int_{\Omega} \boldsymbol{\varepsilon}(v): \delta_{c_{i}} \boldsymbol{\sigma}_{n+1} \mathrm{~d} \Omega-\int_{\Omega} \mathbf{v} \frac{\partial \mathbf{f}_{n+1}}{\partial c_{i}} \mathrm{~d} \Omega-\int_{\Gamma_{t}} \mathbf{v} \frac{\partial \mathbf{t}_{n+1}^{d}}{\partial c_{i}} \mathrm{~d} \Gamma=0 \quad \forall \mathbf{v} \in \mathscr{W}
\end{gathered}
$$

and the constitutive law on the sensitivity described by Equation (15) mentioned above; together with the following initial conditions at time $t=0$,

$$
\begin{gathered}
\delta_{c_{i}} \boldsymbol{\sigma}=\frac{\partial \boldsymbol{\sigma}_{0}}{\partial c_{i}} \\
\delta_{c_{i}} \mathbf{u}=0
\end{gathered}
$$


$\mathscr{W}$ is the space of admissible displacements defined by

$$
\mathscr{W}=\left\{\delta_{c_{i}} \mathbf{u} \in \mathbf{H}^{1}(\Omega) \mid \delta_{c_{i}} \mathbf{u}_{n+1}=\frac{\partial \mathbf{u}_{n+1}^{d}}{\partial c_{i}} \text { on } \Gamma_{\mathbf{u}}\right\}
$$

\subsection{Incremental form of $\mathscr{P}^{s}$}

To see the structure of the computation which is performed step by step, let us denote

$$
\delta_{c_{i}} \mathbf{u}_{n+1}=\delta_{c_{i}} \Delta \mathbf{u}+\delta_{c_{i}} \mathbf{u}_{n}
$$

Therefore, the weak incremental formulation is

$$
\begin{aligned}
& \text { Find } \delta_{c_{i}} \Delta \mathbf{u} \in \mathscr{W}=\left\{\delta_{c_{i}} \Delta \mathbf{u} \in \mathbf{H}^{-1} \mid \delta_{c_{i}} \Delta \mathbf{u}=\frac{\partial \Delta \mathbf{u}^{d}}{\partial c_{i}} \text { on } \Gamma_{u}\right\} \\
& \quad \text { and } \delta_{c_{i}} \boldsymbol{\sigma}_{n+1} \text { satisfying: } \\
& \int_{\Omega} \boldsymbol{\varepsilon}(\mathbf{v}): \boldsymbol{\Xi}_{n+1}: \boldsymbol{\varepsilon}\left(\delta_{c_{i}} \Delta \mathbf{u}\right) \mathrm{d} \Omega \\
& =\int_{\Omega} \mathbf{v} \frac{\partial \mathbf{f}_{n+1}}{\partial c_{i}} \mathrm{~d} \Omega+\int_{\Gamma_{T}} \mathbf{v} \frac{\partial \mathbf{t}_{n+1}^{d}}{\partial c_{i}} \mathrm{~d} \Gamma \\
& +\int_{\Omega} \boldsymbol{\varepsilon}(\mathbf{v}): \boldsymbol{\Xi}_{n+1}: \frac{\partial^{2} \phi\left(\boldsymbol{\sigma}_{n+1}\left(c_{i}\right), c_{i}\right)}{\partial \boldsymbol{\sigma} \partial c_{i}} \Delta t_{n+1} \mathrm{~d} \Omega \\
& -\int_{\Omega} \boldsymbol{\varepsilon}(\mathbf{v}): \mathbf{\Xi}_{n+1}: \mathbf{C}^{-1}: \frac{\partial \mathbf{C}}{\partial c_{i}}:\left(\boldsymbol{\varepsilon}(\Delta \mathbf{u})-\Delta t_{n+1} \frac{\partial \phi\left(\boldsymbol{\sigma}_{n+1}\right)}{\partial \boldsymbol{\sigma}}\right) \mathrm{d} \Omega \\
& -\int_{\Omega} \boldsymbol{\varepsilon}(\mathbf{v}): \boldsymbol{\Xi}_{n+1}: \mathbf{C}^{-1}: \delta_{c_{i}} \boldsymbol{\sigma}_{n} \mathrm{~d} \Omega \quad \forall \mathbf{v} \in \mathscr{V}
\end{aligned}
$$

with the following constitutive law:

$$
\begin{aligned}
\delta_{c_{i}} \boldsymbol{\sigma}_{n+1}= & \boldsymbol{\Xi}_{n+1}: \boldsymbol{\varepsilon}\left(\delta_{c_{i}} \Delta \mathbf{u}\right) \\
& +\boldsymbol{\Xi}_{n+1}:\left[\mathbf{C}^{-\mathbf{1}}: \delta_{c_{i}} \boldsymbol{\sigma}_{n}+\mathbf{C}^{-\mathbf{1}}: \frac{\partial \mathbf{C}}{\partial c_{i}}:\left(\boldsymbol{\varepsilon}(\Delta \mathbf{u})-\Delta t_{n+1} \frac{\partial \phi\left(\boldsymbol{\sigma}_{n+1}\right)}{\partial \boldsymbol{\sigma}}\right)\right] \\
& -\boldsymbol{\Xi}_{n+1}: \frac{\partial^{2} \phi\left(\boldsymbol{\sigma}_{n+1}\left(c_{i}\right), c_{i}\right)}{\partial \boldsymbol{\sigma} \partial c_{i}} \Delta t_{n+1}
\end{aligned}
$$

and the initial conditions already mentioned in Equation (18).

In the case where $c_{i}$ is a constitutive parameter, the initial stresses and the imposed boundary conditions do not depend on $c_{i}$. Therefore, we have

$$
\begin{array}{cc}
\frac{\partial \boldsymbol{\sigma}_{\mathbf{0}}}{\partial c_{i}}=\mathbf{0}, & \frac{\partial \mathbf{f}_{n+1}}{\partial c_{i}}=\mathbf{0} \\
\frac{\partial \mathbf{t}_{n+1}^{d}}{\partial c_{i}}=\mathbf{0}, & \frac{\partial \mathbf{u}_{n+1}^{d}}{\partial c_{i}}=\mathbf{0}
\end{array}
$$

The cost functional $\mathscr{J}$ and its gradient $\nabla \mathscr{J}$ are computed from the solutions of the direct problem $\mathscr{P}$ and of the sensitivities problems $\mathscr{P}^{S}$ using Equations (11) and (12). 


\section{THE FINITE ELEMENT PROGRAMMING}

The direct and sensitivity problem have been programmed in the object oriented finite element code CAST3M [24].

The direct problem has been solved using a radial return mapping for the plastic integration already implemented in the code (functions ecoulement, pasapas). The sensitivity problem has been solved including a programmed sequence at the end of each time step (using the perso facility of the pasapas operator). Therefore, space and time discretization are the same for the direct and auxiliary problems as specified in Reference [15]. A simple scheme of a time step computation is displayed in Figure 1.

At the end of each time step, when convergence of the direct problem is achieved, the stiffness matrix of the direct problem is the consistent tangent operator given in Equation (16). It can therefore be directly used in solving the sensitivity problems. At this point only the force terms arising in the sensitivity analysis have to be computed from already known fields (left-hand side of Equation (21)). If the stiffness matrix is stored in the LU decomposition, computations are fast. For the four sensitivities corresponding to the four parameters to be identified, the complete sensitivity analysis represented approximately half of the computational burden of the direct problem. This performance can still be enhanced provided that less fields are stored during the computation and that the time steps are optimized. This was however not a main objective of this work.

Let us now discuss shortly an important detail related to the set-up of the lining. This procedure introduces a change of the domain as previously explained. The initial assumption is that one can consider the direct problem on the whole domain: lining + rock mass and neglect the influence of the lining using a vanishing Young's modulus before the set-up of the lining. Theoretically, as all expressions are obtained between time steps on a fixed domain, the set-up of the support does not influence the deduction of the sensitivity problem.

From a numerical point of view, the numerical value chosen for the vanishing Young's modulus did not influence the result of the direct problem. However for the computations of the sensitivities, we experienced that if the vanishing Young's modulus is chosen too small, i.e. $E_{\text {lining }} \ll 10^{-15} E_{\text {rock }}$, important errors are created at the first time step after the set up of the lining. These propagate in the sequel perturbing the solution. This can be explained by floating point errors in the algebraic manipulation in the left-hand side of Equation (21).

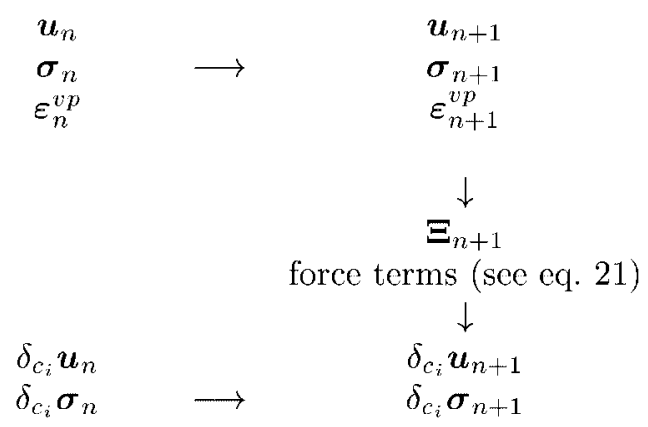

Figure 1. Scheme of a time step for the direct and sensitivity problem. 
A Levenberg-Marquardt [25] algorithm has been used for the minimization procedure and programmed using the numerical package SCILAB developed at INRIA [26]. It is important to point out that the use of a singular value decomposition was necessary in the LevenbergMarquardt algorithm in order to assure a robust estimate of the minimization step.

The computational time for the direct and sensitivity problem was less than 5 min for 1 iteration of the minimization algorithm (direct computation and direct differentiation problems) in a problem with 4460 degrees of freedom on a Intel PentiumIII machine with $550 \mathrm{MHz}$ and 256 MB RAM running under Linux.

\section{RESULTS AND DISCUSSION}

In this section, we shall present a series of identifications based on simulated measurements. The 'real' parameters $\mathbf{c}_{\text {real }}$, to be identified in the sequel, have been chosen in order to reproduce the order of magnitude found in situ and to be compatible with available experimental data on deep argillaceous rocks of Eastern France [27].

The first example presented corresponds to a one-dimensional problem with the following 'real' parameters:

$$
E=4 \mathrm{GPa}, \quad v=0.3, \quad \sigma_{Y}=1 \mathrm{MPa}, \quad N=8, \quad K=140 \mathrm{MPa} \mathrm{s}^{1 / N}
$$

and an isotropic initial stress state defined by

$$
\boldsymbol{\sigma}_{\mathbf{0}}=-12 \mathbf{I} \mathrm{MPa}
$$

where I denotes the second rank identity tensor.

The second example corresponds to a two-dimensional section of a tunnel, with:

$$
E=4 \mathrm{GPa}, \quad v=0.3, \quad \sigma_{Y}=3 \mathrm{MPa} \quad N=8, \quad K=120 \mathrm{MPa} \mathrm{s}^{1 / N}
$$

and an anisotropic initial stress state:

$$
\begin{aligned}
& \boldsymbol{\sigma}_{0}=\left(\begin{array}{ccc}
\sigma_{\text {Horizontal }} & 0 & 0 \\
0 & \sigma_{\text {Vertical }} & 0 \\
0 & 0 & \sigma_{\text {horizontal }}
\end{array}\right) \\
& \sigma_{\text {Vertical }}=-12 \mathrm{MPa} \quad \sigma_{\text {Horizontal }}=\sigma_{\text {horizontal }}=-9.6 \mathrm{MPa}
\end{aligned}
$$

The lining is supposed to have an elastic behaviour with a Young's Modulus of $15 \mathrm{GPa}$ and a Poisson's ratio of 0.25 corresponding to a reinforced concrete.

In both examples, we consider that displacements and contact forces are available as measurements at different locations on the lining and in the rock mass (see Figure 9).

For the identification problem, we have considered a cost functional $\mathscr{J}$ as defined in (11). The gradient $\nabla \mathscr{J}$ has been computed using (12). The weights $w_{u_{i j}}$ and $w_{t_{i j}}$ were considered equal for all measurements locations and were just adjusted to obtain a similar weight between the error in displacement and the error in force. The minimization procedure was performed using a preconditioning as explained in Reference [25]. As a consequence $\|\nabla \mathscr{J}\|$ represents the euclidian norm of the preconditioned gradient of the cost functional. 


\subsection{One-dimensional example}

Let us consider the simple case of a circular tunnel of radius $R$ in an infinite medium with isotropic initial stresses conducting to a one-dimensional problem, in the lined and in the unlined case.

As mentioned before, the time of excavation is supposed to be negligible compared with the time of interest: the excavation is completed at $t=0^{+}$. The lining, in the case of the supported cavity, is supposed to be set up approximately 20 days after the end of the excavation.

The measurements for the unlined tunnel are radial displacements at three different points in the rock mass: at the tunnel wall $R$, at $2 R$ and, respectively, at $3 R$ from the tunnel wall.

The measurements for the lined tunnel are convergence (radial displacements at the tunnel wall) and the contact force at the interface between the lining and the rock mass.

In all cases the optimal value of parameters $\left(\mathbf{c}_{\text {real }}\right)$ has been recovered after 10-20 iterations with an almost vanishing cost functional. This means that the final simulated results match the measurements as illustrated in Figure 3 where 10\% noise has been added to measurements.

A series of identified values for different starting points as well as the decrease of the value of the cost functional and of its gradient are represented in Tables I, II and III.

The number of measurement locations involved in the identification was not very important, similar results have been obtained with 1 or 3 sets of displacement data.

The representation of the radial distribution of the sensitivities (see Figures 2 and 5) can be linked to the 'information content' of the mechanical fields with respect to the considered

Table I. Results of the identification in the one-dimensional unlined case with no added noise.

\begin{tabular}{lccccccc}
\hline & $E(\mathrm{GPa})$ & $\sigma_{Y}(\mathrm{MPa})$ & $N$ & $K\left(\mathrm{MPa} \mathrm{s}^{-1 / N}\right)$ & $\mathscr{J}$ & $\|\nabla \mathscr{J}\|$ & Iterations \\
\hline Initial & 1 & 1 & 9 & 100 & 0.129 & 0.199 & \\
Final & 4.132 & 1.439 & 7.926 & 130.0 & $2.3 \times 10^{-6}$ & $1 \times 10^{-5}$ & 10 \\
Initial & 5 & 1 & 7 & 200 & $1.7 \times 10^{-4}$ & $3.7 \times 10^{-3}$ & \\
Final & 4.12 & 1.982 & 7.245 & 157.8 & $1 \times 10^{-7}$ & $8.9 \times 10^{-6}$ & 12 \\
Intel & 4 & 0.5 & 7.5 & 100 & 0.250 & 0.348 & \\
Final & 3.97 & 1.048 & 8.032 & 135.5 & $2.9 \times 10^{-6}$ & $6.4 \times 10^{-5}$ & 15 \\
Initial & 4.50 & 1 & 7.5 & 160 & $2.3 \times 10^{-5}$ & $2.1 \times 10^{-4}$ & \\
Final & 4.127 & 1.665 & 7.64 & 140 & $2.1 \times 10^{-6}$ & $7.0 \times 10^{-6}$ & 12 \\
\hline Real & 4 & 1 & 8 & 140 & & & \\
\hline
\end{tabular}

Table II. Results of the identification in the one-dimensional lined case with no added noise.

\begin{tabular}{lccccccc}
\hline & $E(\mathrm{GPa})$ & $\sigma_{Y}(\mathrm{MPa})$ & $N$ & $K\left(\mathrm{MPa} \mathrm{s}^{-1 / N}\right)$ & $\mathscr{J}$ & $\|\nabla \mathscr{J}\|$ & Iterations \\
\hline Initial & 1 & 1 & 9 & 100 & 790.13 & 635.6 & \\
Final & 4.014 & 0.908 & 8.129 & 135.5 & $3.5 \times 10^{-5}$ & $1 \times 10^{-2}$ & 14 \\
Initial & 1 & 1 & 7 & 200 & 714.22 & 399.3 & \\
Final & 4.00 & 1.060 & 7.947 & 142.0 & $1.4 \times 10^{-5}$ & $9 \times 10^{-3}$ & 12 \\
Initial & 4 & 0.5 & 7.5 & 100 & 250.9 & 183.1 & \\
Final & 4.0 & 1.02 & 7.98 & 140.7 & $2.7 \times 10^{-6}$ & $4.7 \times 10^{-3}$ & 15 \\
Initial & 4.50 & 1 & 7.5 & 160 & 0.685 & 0.78 & \\
Final & 4.00 & 1.05 & 7.95 & 141.7 & $1.06 \times 10^{-5}$ & $7.6 \times 10^{-3}$ & 13 \\
\hline Real & 4 & 1 & 8 & 140 & & & \\
\hline
\end{tabular}


Table III. Results of the identification in the one-dimensional lined case with additional $10 \%$ noise.

\begin{tabular}{lccccccc}
\hline & $E(\mathrm{GPa})$ & $\sigma_{Y}(\mathrm{MPa})$ & $N$ & $K\left(\mathrm{MPa} \mathrm{s}^{-1 / N}\right)$ & $\mathscr{J}$ & $\|\nabla \mathscr{J}\|$ & Iterations \\
\hline Initial & 1 & 1 & 9 & 100 & 792.6562 & 649.4 & \\
Final & 4.213 & 2.144 & 7.30 & 151.1 & 0.7914 & $7.0892 \times 10^{-3}$ & 10 \\
Initial & 5 & 1 & 7 & 200 & 4.057 & 6.05 & \\
Final & 4.199 & 2.21 & 7.21 & 156.0 & 0.7912 & $4.1716 \times 10^{-3}$ & 9 \\
Initial & 4 & 0.5 & 7.5 & 100 & 254.86 & 180.4 & \\
Final & 4.176 & 2.339 & 7.045 & 165.5 & 0.7913 & $5.96 \times 10^{-3}$ & 11 \\
Initial & 4.50 & 1 & 7.5 & 160 & 1.822 & 1.157 & \\
Final & 4.184 & 2.293 & 7.10 & 162.1 & 0.7910446 & $3.2713 \times 10^{-3}$ & 11 \\
\hline Real & 4 & 1 & 8 & 140 & & & \\
\hline
\end{tabular}

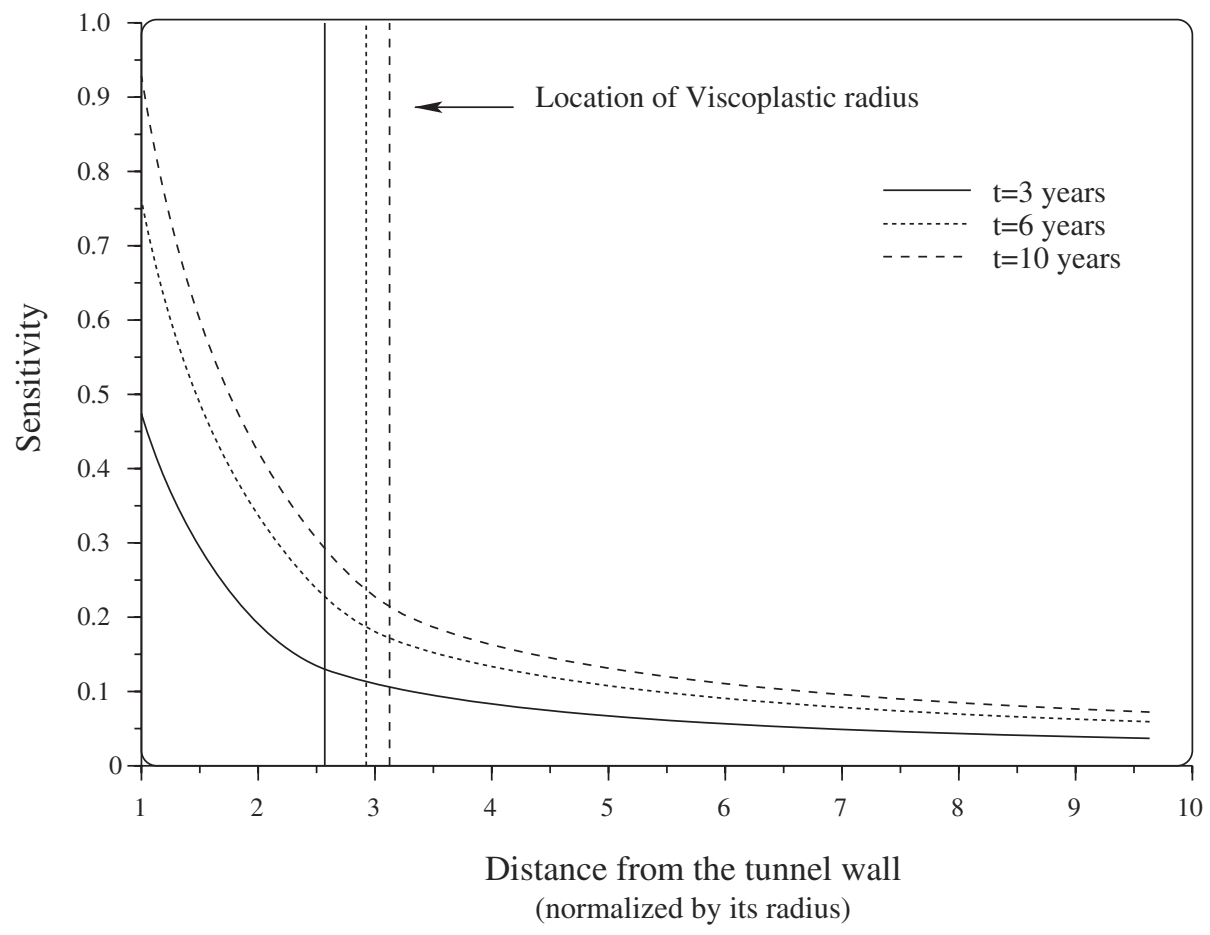

Figure 2. Sensitivity on radial displacement $\delta_{N} u_{r} / R$, unlined tunnel (one-dimensional).

parameter. Thus it permits to decide the best-measurement locations and observation periods for the identification.

Figure 2 shows that the strongest sensitivities on the displacements are contained in a region within $3 R$ which corresponds to the viscoplastic zone. The sensitivity on the radial stress (see Figure 5) is also concentrated in the viscoplastic zone, but the sensitivity maximum is located this time near the viscoplastic border in the rock mass.

The sensitivities are time increasing. However, for the lined tunnel (Figure 4), the sensitivities reach an almost constant value with time. The time evolution of the sensitivities show that the 


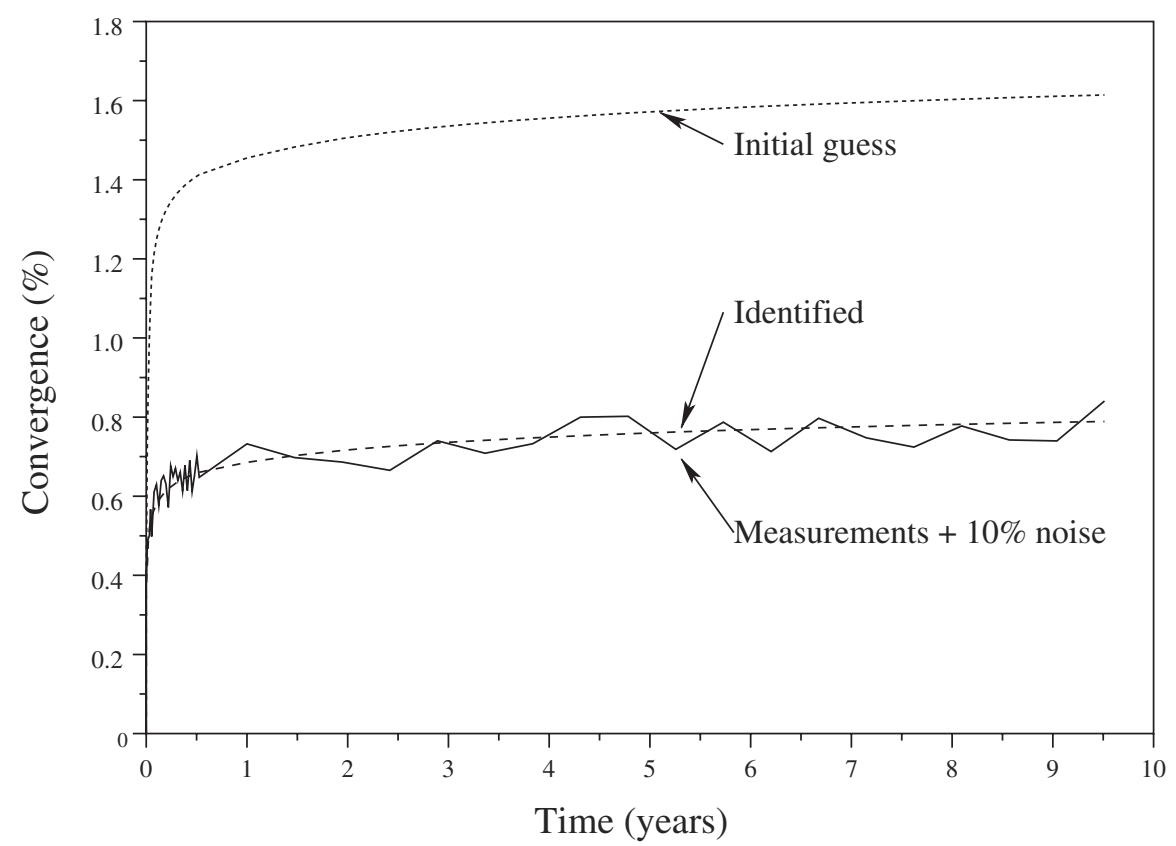

Figure 3. Fit of convergence data $u / R$, one-dimensional lined tunnel with $10 \%$ noise.

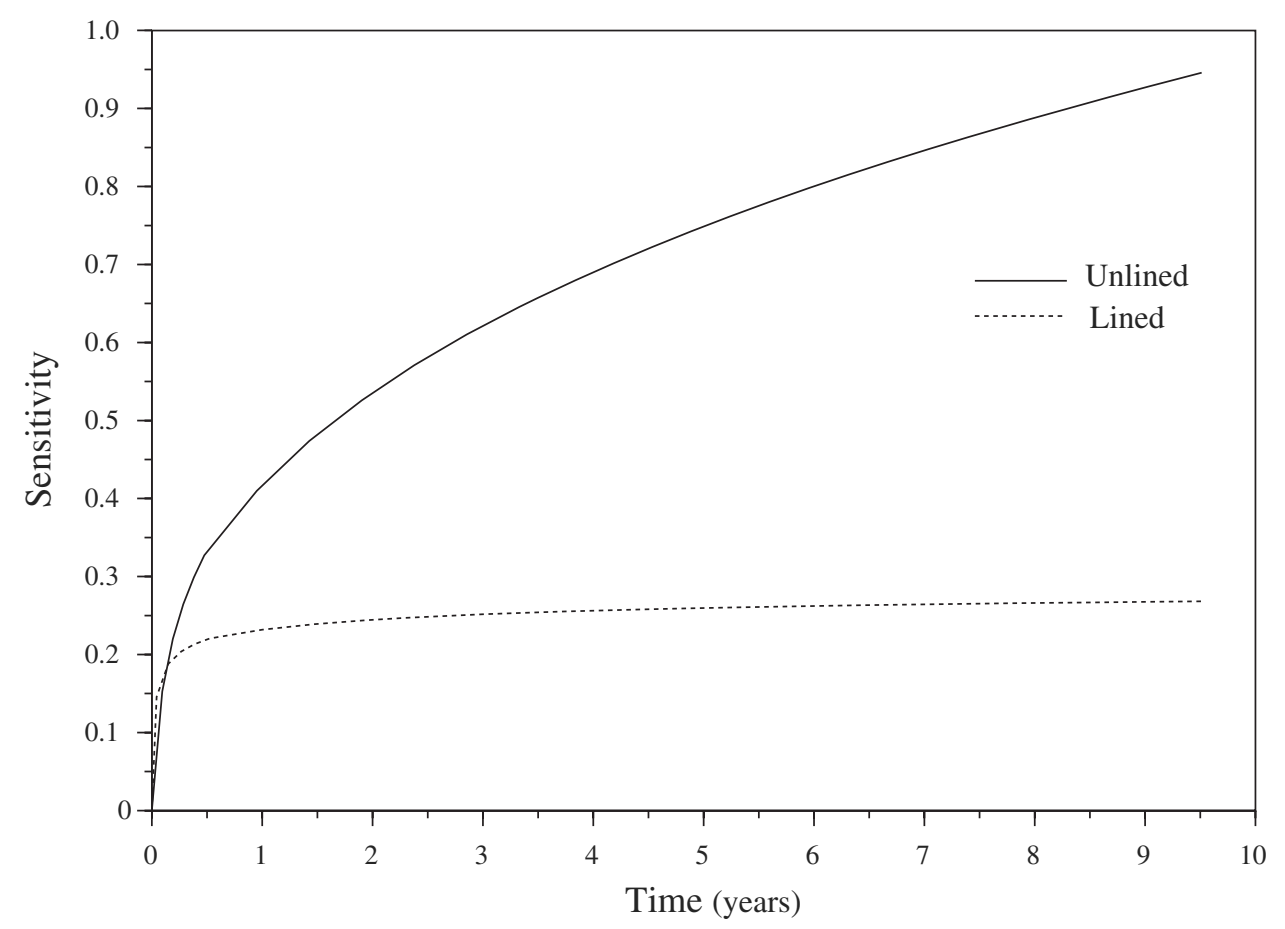

Figure 4. Evolution of $\delta_{N} u_{r} / R$ with time in the one-dimensional lined and unlined cases. 


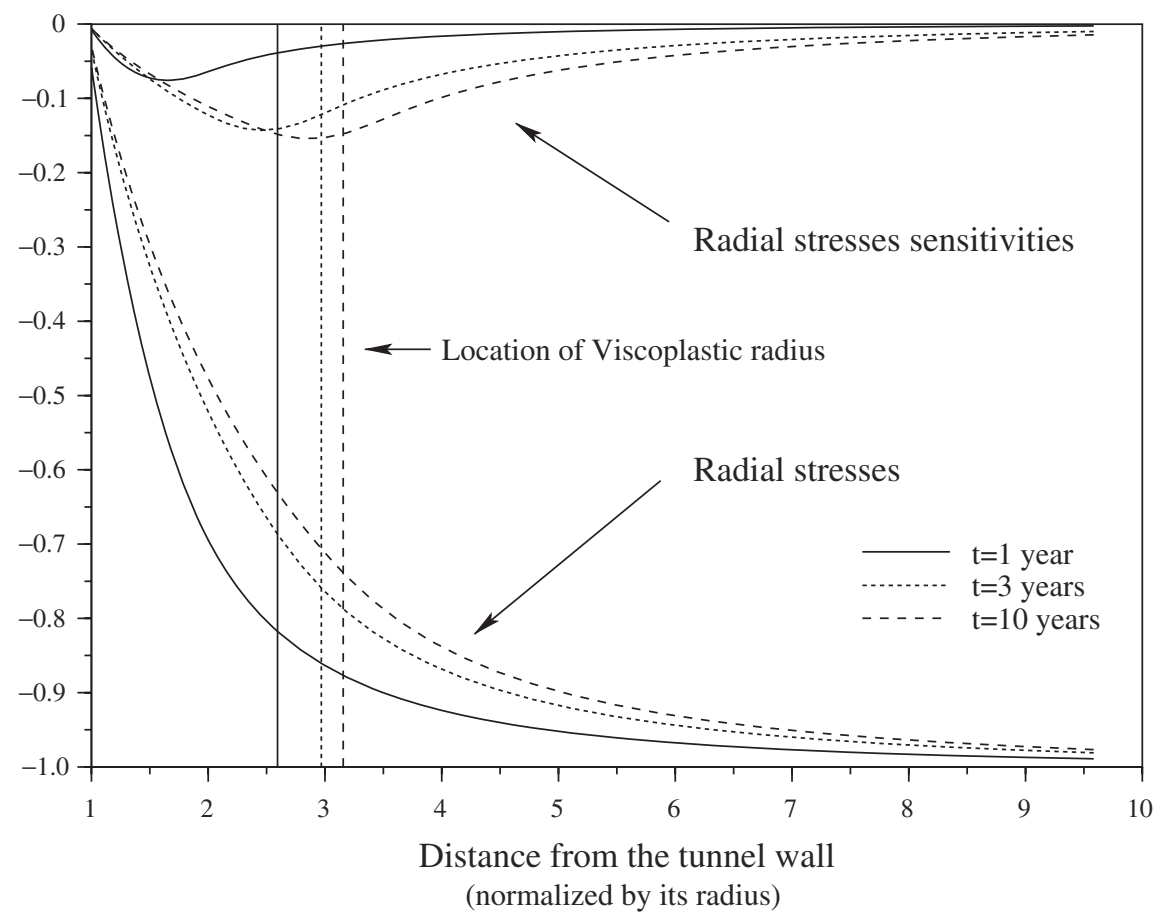

Figure 5. Evolution of $\sigma_{r r} / \sigma_{0}$ and $\delta_{N} \sigma_{r r} / \sigma_{0}$ function of $r$ in the one-dimensional unlined tunnel case.

Table IV. Influence of the observation period on the identification, one-dimensional case, unlined tunnel. Same initial guess $\left(E=1 \mathrm{GPa}, \sigma_{Y}=1 \mathrm{MPa}, N=7, K=200 \mathrm{MPa} \mathrm{s}^{1 / N}\right)$.

\begin{tabular}{cccccc}
\hline$t_{\text {start }}$ & $t_{f}$ & $E(\mathrm{GPa})$ & $\sigma_{Y}(\mathrm{MPa})$ & $N$ & $K\left(\mathrm{MPa} \mathrm{s}^{-1 / N}\right)$ \\
\hline 0 & 10 years & 4.004 & 1.06 & 7.947 & 142.0 \\
0 & 3 year & 4.003 & 1.94 & 7.201 & 170.1 \\
0 & 1 year & 3.99 & 2.16 & 7.09 & 189.5 \\
\hline
\end{tabular}

lined and unlined problems have different characteristic times. This can be explained by the presence of a relatively stiff lining when compared with the rock mass $\left(E_{\text {rock }} / E_{\text {lining }} \approx 0.25\right)$ and has already been interpreted from a geotechnical point of view by Bérest and Nguyen Minh [28].

The increase of the length of the observation period enhances, as expected, the accuracy of the identified parameters (see Table IV).

Results for lined tunnels are generally more accurate (see Table II). In this case the observation period is long enough for the structure to reach the steady state (Figure 4) and contains therefore all the possible information. For unlined tunnels, the observation period is not long enough and this can explain the poor recovery of the optimum parameters in this case (see Table I).

As a final remark, let us notice that the global shape of the sensitivity is similar for all constitutive parameters. 


\subsection{Two-dimensional case}

In the two-dimensional case, the geometry of the tunnel and the corresponding finite element mesh as well as the location of the measurement are represented in Figure 9.

In this case an anisotropic initial stress with $K_{0}=0.8$ has been considered. The excavation and lining sequence is the same as in the one-dimensional case.

Like in the one-dimensional case, the values have been recovered after a small number of iterations with an almost vanishing cost functional (see Tables V and VI). A convergence example of the parameters towards their 'real value' is displayed in Figure 6 in the case where the data were perturbed by a white noise of $10 \%$.

The maximum spatial distribution of the sensitivity is located as in the one-dimensional case, near the tunnel wall in the viscoplastic region (see Figure 8). The sensitivity of radial stresses on the upper-vertical and horizontal radius (see Figures 7 and 8) show profiles which are similar to the ones obtained for the one dimensional case (see Figure 5).

The exact distribution of other components of the stresses will depend on the tunnel geometry and on the initial stress state. The distribution of the Von Mises stress as well as the Von Mises norm of the sensitivity stress tensor are displayed in Figure 8. One can remark a concentration around the diagonal directions which should be related with the ratio between the initial vertical

Table V. Results of the identification in the two-dimensional case.

\begin{tabular}{lccccccc}
\hline & $E(\mathrm{GPa})$ & $\sigma_{Y}(\mathrm{MPa})$ & $N$ & $K\left(\mathrm{MPa} \mathrm{s}^{-1 / N}\right)$ & $\mathscr{J}$ & $\|\nabla \mathscr{J}\|$ & Iterations \\
\hline Initial & 1 & 2.0 & 7 & 200 & 1713.2 & 855.3 & \\
Final & 4.00 & 2.995 & 8.00 & 119.8 & $1 \times 10^{-7}$ & $1.12 \times 10^{-3}$ & 12 \\
Initial & 6 & 2 & 9 & 50 & 90.48 & 427.3 & \\
Final & 3.998 & 3.126 & 7.84 & 124.7 & $4.06 \times 10^{-5}$ & $2.03 \times 10^{-3}$ & 15 \\
Initial & 6 & 4 & 7 & 200 & 49.63 & 80.14 & \\
Final & 3.999 & 3.04 & 7.94 & 121.5 & $2.27 \times 10^{-5}$ & $8.9 \times 10^{-2}$ & 15 \\
Initial & 1 & 2 & 9 & 200 & 1083.2 & 908.2 & \\
Final & 4.00 & 3.0 & 7.998 & 120.1 & $1 \times 10^{-7}$ & $6.0 \times 10^{-3}$ & 11 \\
\hline Real & 4.00 & 3.0 & 8 & 120 & & & \\
\hline
\end{tabular}

Table VI. Results of the identification in the two-dimensional case. No measurements inside the rock mass.

\begin{tabular}{lccccccc}
\hline & $E(\mathrm{GPa})$ & $\sigma_{Y}(\mathrm{MPa})$ & $N$ & $K\left(\mathrm{MPa} \mathrm{s}^{-1 / N}\right)$ & $\mathscr{J}$ & $\|\nabla \mathscr{J}\|$ & Iterations \\
\hline Initial & 1 & 2.0 & 7 & 200 & 1604.80 & 800.7 & \\
Final & 4.0 & 3.00 & 7.999 & 120.00 & $<1 \times 10^{-7}$ & $5.9 \times 10^{-4}$ & 12 \\
Initial & 6 & 2 & 9 & 50 & 85.04 & 424.32 & \\
Final & 4.00 & 2.997 & 8.001 & 120.00 & $<1 \times 10^{-7}$ & $5.6 \times 10^{-3}$ & 12 \\
Initial & 6 & 4 & 7 & 200 & 46.7 & 71.69 & \\
Final & 4.00 & 3.01 & 7.98 & 120.5 & $1.13 \times 10^{-5}$ & $7.1 \times 10^{-3}$ & 15 \\
Initial & 1 & 2 & 9 & 200 & 1006.2 & 840.42 & \\
Final & 4.00 & 3.001 & 7.999 & 120.1 & $<1 \times 10^{-7}$ & $5.11 \times 10^{-3}$ & 11 \\
\hline Real & 4 & 3 & 8 & 120 & & & \\
\hline
\end{tabular}




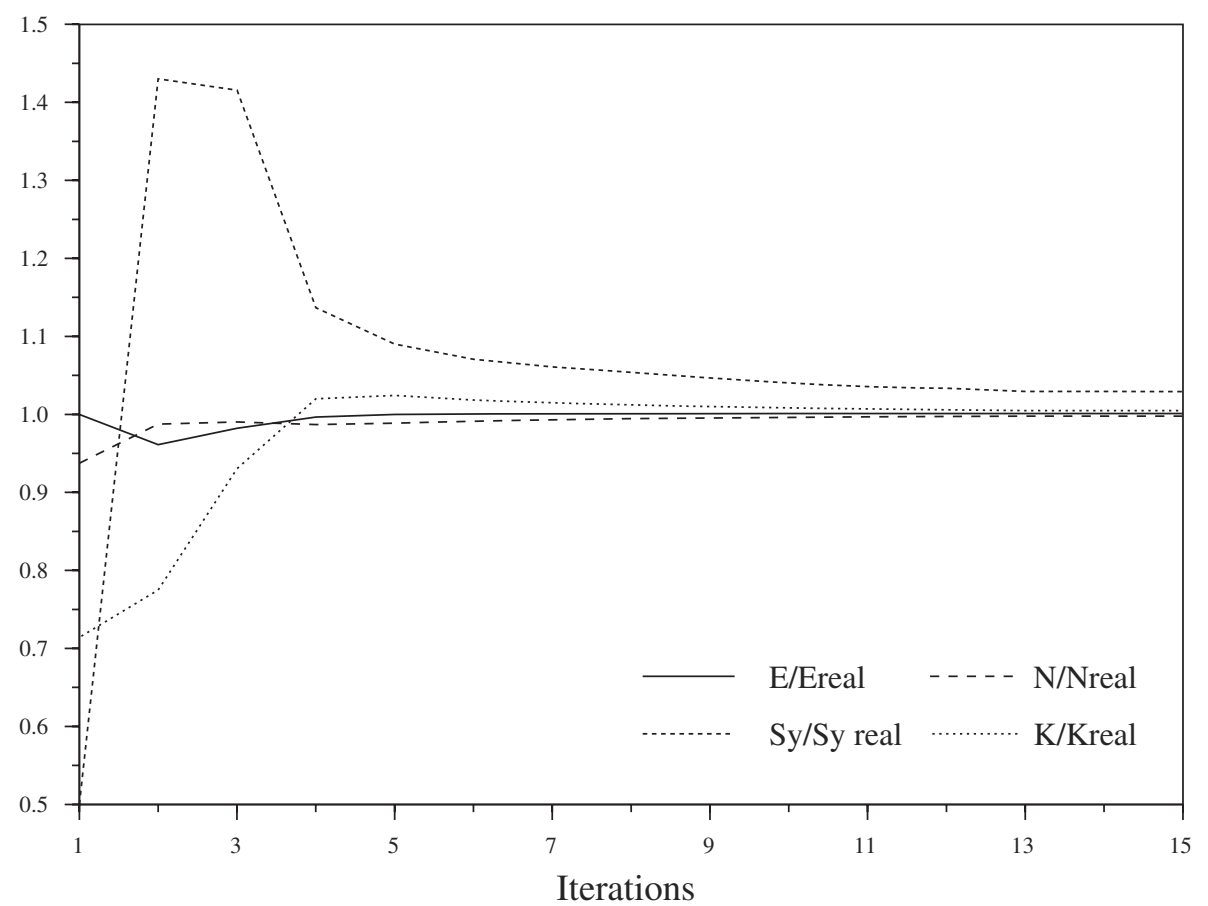

Figure 6. Evolution of the different parameters (scaled by the optimal value) during the LevenbergMarquardt algorithm.

and horizontal stresses. The sensitivity distributions permit to identify the optimal locations for installing the pressure cells, measuring the contact force, and the extensometers.

In the presented computations we have not observed an influence of the number of measurement locations on final results of the optimization. This might astonish, but one has to recall that in the presented computation, sensitivity histories are similar in all locations. In the real case the dispersion of the data comes on the one hand from the noisy measurements and on the other hand from the inhomogeneity of the medium.

\subsection{Uniqueness discussion}

The present results showed that in one- and two-dimensional configurations the parameters of the constitutive law could be reconstructed from contact forces and displacement measurements in the lining or in the rock mass. In all cases, measurements were matched almost perfectly, even if the estimated parameters were still at a certain distance from the real values. The question arising in such a case is that of the uniqueness of this inverse identification problem.

From a practical view, we have been plotting the variation of the cost functional in different planes. The shapes of the functional presented generally long flat valleys surrounding the optimum (see Figure 10) in both lined and unlined cases showing that the optimum is always 


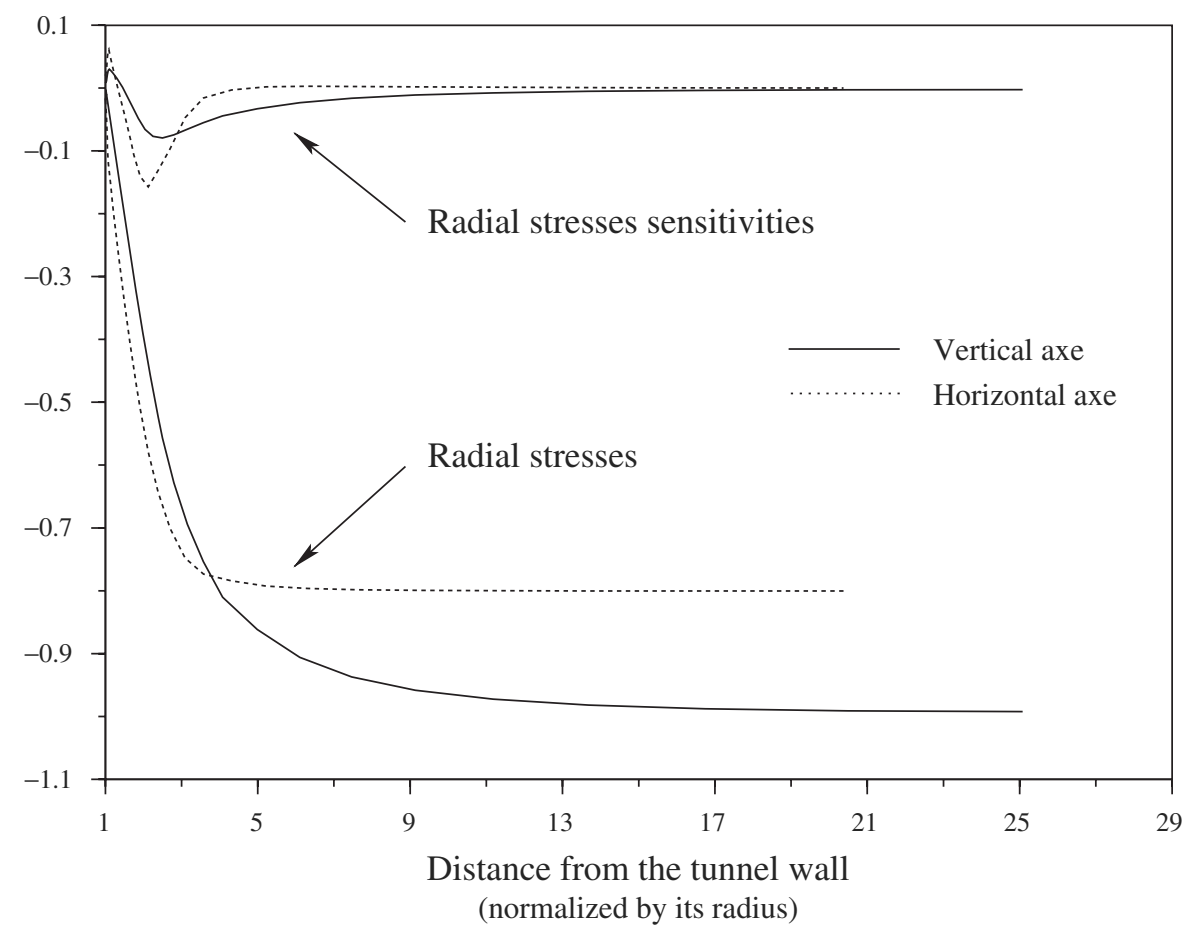

Figure 7. Profile of $\sigma_{r r} / \sigma_{0}$ and $\delta_{N} \sigma_{r r} / \sigma_{0}$ in the two-dimensional case $\left(K_{0}=0.8\right)$.
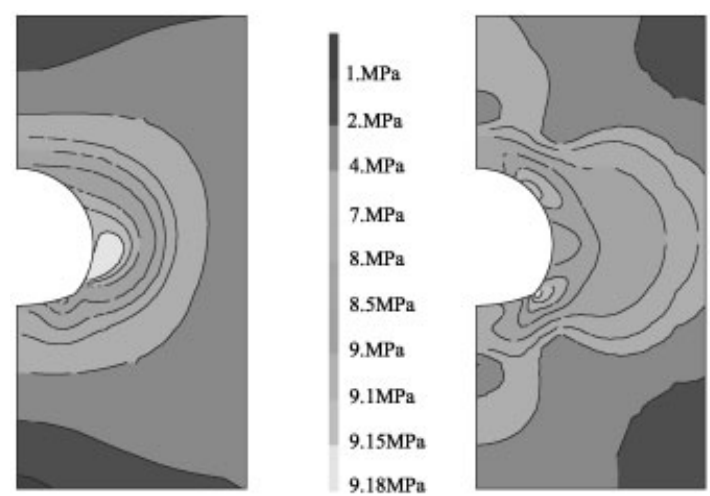
$0.2 \mathrm{MPa}$
$0.3 \mathrm{MPa}$
$0.5 \mathrm{MPa}$
$0.8 \mathrm{MPa}$
1.MPa
2.MPa
3.MPa
4.MPa
5. $\mathrm{MPa}$
6.MPa

Figure 8. Von Mises of $\boldsymbol{\sigma}$ (Yield Limit $3 \mathrm{MPa}$ ) and $\delta_{N} \boldsymbol{\sigma}$.

unique. In Figure 10, we have displayed the shape of the cost functional in the plane $N-K$. The representation in the $\sigma_{Y}-E$ plane is similar.

The variations of the cost functional in this area are generally small and interfere with numerical noise. Similar behaviour has already been reported in Reference [14]. 


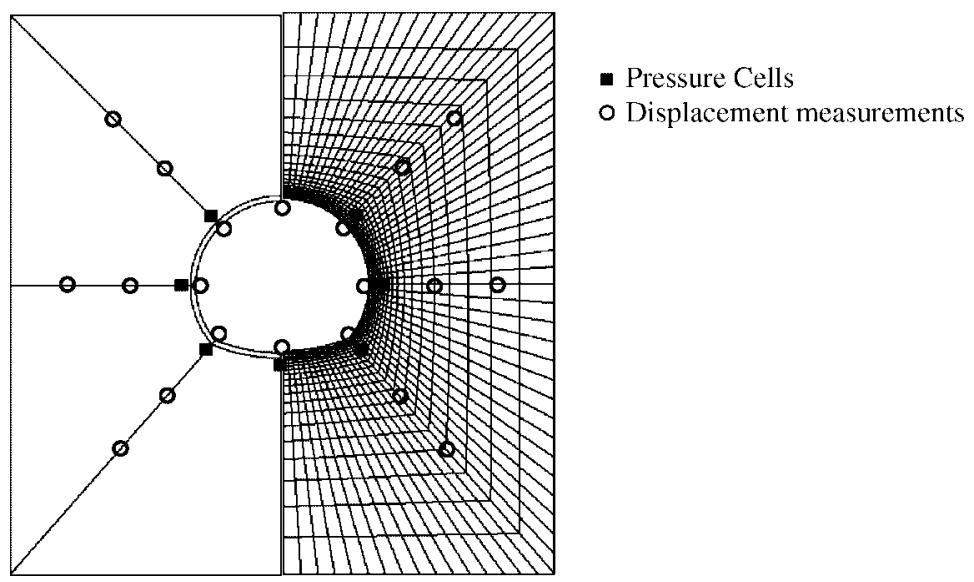

Figure 9. Close view of the finite element mesh. Location of measurements points.

The uniqueness observation could be related to a series of uniqueness proofs recently obtained in viscoelasticity [29, 30] for simple configurations. These mathematical results are obtained using an integrodifferential representation of the viscoelastic constitutive law and further work is needed to extend these results to the exact form of elastoviscoplasticity used here. The flat valley is to be related to a bad stability of the cost functional. Therefore, one should probably investigate other forms of cost functionals providing a better sensitivity with respect to the parameters.

\section{CONCLUSION}

The present paper discussed the identification of constitutive parameters for an elastoviscoplastic law. The identification is based on a minimization algorithm using a gradient computed by direct differentiation. The constitutive parameters of the rock mass around an underground lined or unlined tunnel were obtained from simulated measurements, without using any a priori information or regularization technique.

The results show that the direct differentiation method can be successfully applied in the particular case of tunneling. The sudden change of configuration given by the installation of the lining and the subsequent modelling has not posed important difficulties.

The obtained sensitivities also permit the optimization of the location of instrumentation in the rock mass as well as of the observation period. This is of particular interest in the context of in situ monitoring of underground structures.

The probabilistic character of measurements data and of rock mass parameters can be included in the present method by adding covariance matrices in the optimization process as explained in Reference [7].

Further studies should include a larger range of constitutive equations and data coming from field measurements. Some preliminary results are reported in Reference [31]. 

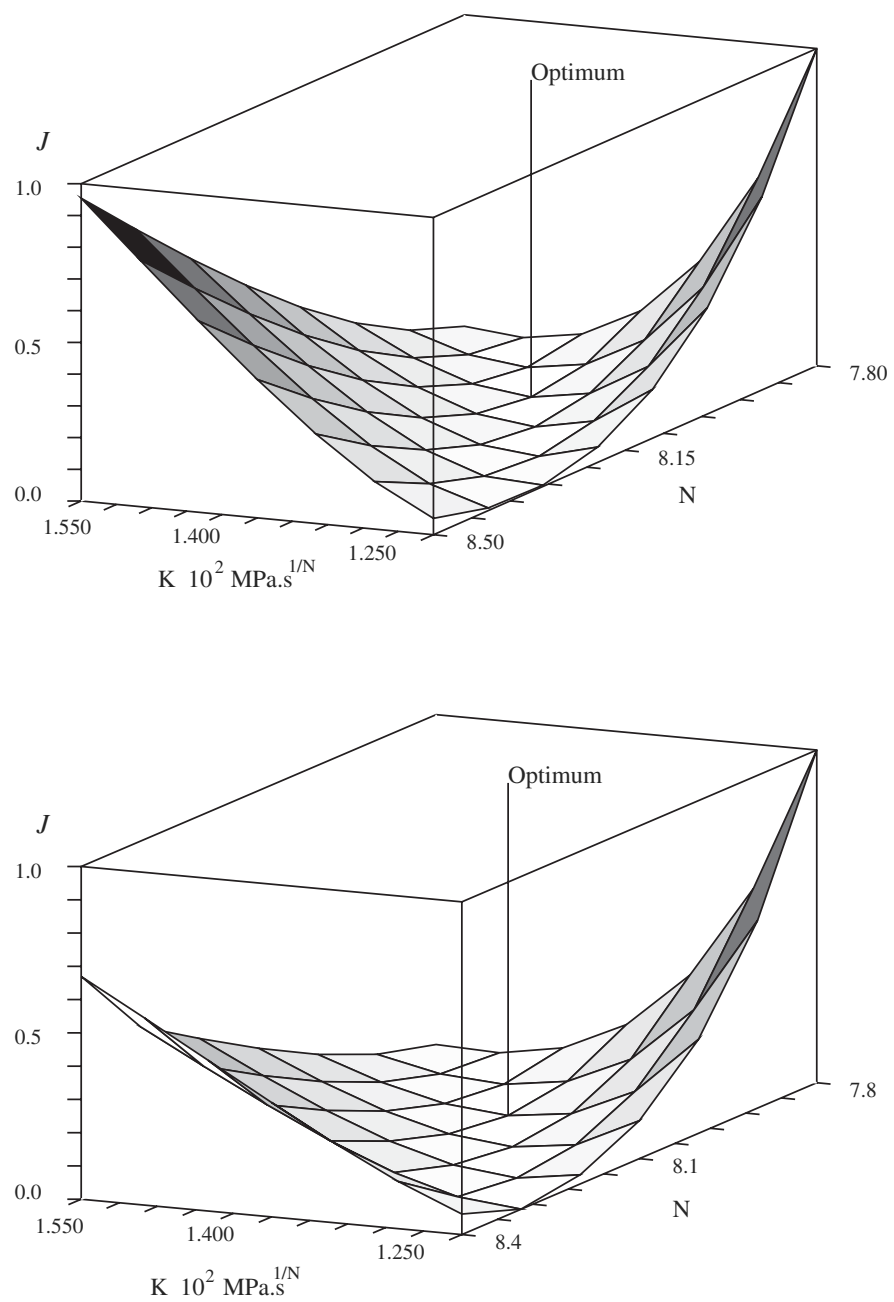

Figure 10. Three-dimensional plot of the cost function in the plane $N-K$ for the one-dimensional lined and unlined cases.

\section{ACKNOWLEDGEMENTS}

This study is part of an ongoing research project sponsored by the French Nuclear Agency for Radioactive Waste Management (ANDRA), their support is gratefully acknowledged.

\section{APPENDIX: DERIVATION OF THE SENSITIVITIES CONSTITUTIVE LAW}

After eliminating $\delta_{c_{i}} \varepsilon_{n+1}^{\mathrm{vp}}$ in Equation (13) using (14), we obtain

$$
\delta_{c_{i}} \boldsymbol{\sigma}_{n+1}=\mathbf{C}:\left(\varepsilon\left(\delta_{c_{i}} \mathbf{u}_{n+1}\right)-\Delta t_{n+1} \frac{\partial^{2} \phi\left(\boldsymbol{\sigma}_{n+1}\right)}{\partial \boldsymbol{\sigma}^{2}} \delta_{c_{i}} \boldsymbol{\sigma}_{n+1}\right)
$$




$$
\begin{aligned}
& -\mathbf{C}:\left(\delta_{c_{i}} \boldsymbol{\varepsilon}_{n}^{\mathrm{vp}}+\Delta t_{n+1} \frac{\partial^{2} \phi\left(\boldsymbol{\sigma}\left(c_{i}\right), c_{i}\right)}{\partial \boldsymbol{\sigma} \partial c_{i}}\right) \\
& +\frac{\partial \mathbf{C}}{\partial c_{i}}:\left(\boldsymbol{\varepsilon}\left(\mathbf{u}_{n+1}\right)-\boldsymbol{\varepsilon}_{n+1}^{\mathrm{vp}}\right)+\frac{\partial \boldsymbol{\sigma}_{0}}{\partial c_{i}}
\end{aligned}
$$

Expressing $\delta_{c_{i}} \boldsymbol{\sigma}_{n+1}$ as in functions of $\boldsymbol{\varepsilon}\left(\delta_{c_{i}} \mathbf{u}_{n+1}\right)$, we get

$$
\begin{aligned}
\underbrace{\left(\mathbf{C}^{-1}+\Delta t_{n+1} \frac{\partial^{2} \phi\left(\boldsymbol{\sigma}_{n+1}\right)}{\partial \boldsymbol{\sigma}^{2}} \delta_{c_{i}}\right)}_{\mathbf{\Xi}_{n+1}^{-1}}: \delta_{c_{i}} \boldsymbol{\sigma}_{n+1}= & \boldsymbol{\varepsilon}\left(\delta_{c_{i}} \mathbf{u}_{n+1}\right) \\
& -\delta_{c_{i}} \boldsymbol{\varepsilon}_{n}^{\mathrm{vp}}+\Delta t_{n+1} \frac{\partial^{2} \phi\left(\boldsymbol{\sigma}\left(c_{i}\right), c_{i}\right)}{\partial \boldsymbol{\sigma} \partial c_{i}} \\
& +\mathbf{C}^{-1}: \frac{\partial \mathbf{C}}{\partial c_{i}}:\left(\boldsymbol{\varepsilon}\left(\mathbf{u}_{n+1}\right)-\boldsymbol{\varepsilon}_{n+1}^{\mathrm{vp}}\right) \\
& +\mathbf{C}^{-1}: \frac{\partial \boldsymbol{\sigma}_{0}}{\partial c_{i}}
\end{aligned}
$$

Using the definition of the Consistent Tangent Moduli $\boldsymbol{\Xi}_{n+1}(16)$, we easily get Equation (15).

\section{REFERENCES}

1. Nguyen Minh D, Habib P, Guerpillon Y. Time dependent behaviour of a pilot tunnel driven in hard marls. In Design and Performance of underground excavations ISRM/BGS: Cambridge, 1984; 453-459.

2. Nguyen Minh D, Maitournam H, Braham S, Durup JG. A tentative of interpretation of long term subsidence measurements over a solution mined cavern field. In Proceedings of the 7th International Symposium on Salt, vol. I, 1992; 441-449.

3. Biot MA. General theory of three-dimensional consolidation. Journal of Applied Physics 1941; 122:155-164.

4. Bérest P. Viscoplasticity in rock mechanics. In Geomaterials: Constitutive equations and modeling, Darve F (ed.). Elsevier Applied Science: Amsterdam, 1990; 239-262.

5. Cristescu ND, Hunsche U. Time Effects in Rocks Mechanics. Wiley: New York, 1999.

6. Bui HD. Introduction aux Problèmes Inverses en Mécanique des Matériaux. Eyrolles: Paris, 1993.

7. Tarantola A. Inverse Problem Theory. Elsevier: Amsterdam, 1987.

8. Sakurai S. Back analysis in rock engineering. In Comprehensive Rock Engineering, vol. 4, Hudson JA (ed.). Pergamon Press: Oxford, 1993; 543-569.

9. Gioda GC, Locatelli L. Back analysis of the measurements performed during the excavation of a shallow tunnel in sand. International Journal for Numerical and Analytical Methods in Geomechanics 1999; 23(13):1407-1425.

10. Swoboda G, Ichikawa Y, Dong Q, Zaki M. Back analysis of large geotechnical models. International Journal for Numerical and Analytical Methods in Geomechanics 1999; 23(13):1455-1472.

11. Ledesma A, Gens A, Alonso EE. Identification of parameters in geomechanics using a maximum likelihood approach. In IUTAM Symposium on Inverse Problems in Engineering Mechanics, 11-15 May 1992, Tokyo, Japan, Tanaka M, Bui HD (eds). Springer, Verlag: Berlin, 1993.

12. Gioda G, Maier G. Direct search solution of an inverse problem in elastoplasticity: identification of cohesion, friction angle and in situ stress by pressure tunnel tests. International Journal for Numerical Methods in Engineering 1980; 15:182-1848.

13. Tortorelli DA, Michaleris P. Design sensitivity analysis: overview and review. Inverse Problems in Engineering 1994; 1:71-103.

14. Constantinescu A, Tardieu N. On the identification of elastoviscoplastic constitutive law from indentation tests. Inverse Problems in Engineering 2001; 9:19-44.

15. Vidal CA, Lee HS, Haber RB. The consistent tangent operator for design sensitivity analysis of history-dependent response. Computing Systems in Engineering 1991; 2(5/6):509-523.

16. Vidal CA, Haber RB. Design sensitivity analysis for rate-independent elastoplasticity. Computer Methods in Applied Mechanics and Engineering 1993; 107:393-431. 
17. Tsay JJ, Arora JS. Nonlinear structural design sensitivity analysis for path dependent problems. Computer Methods in Applied Mechanics and Engineering 1990; 81:183-228.

18. Galarza GA, Carrera J, Medina A. Computational techniques for optimization of problems involving non-linear transient simulations. International Journal for Numerical Methods in Engineering 1999; 45(3):319-334.

19. Perzyna P. Fundamental problems in viscoplasticity. Advances in Applied Mechanics 1966; 9:243-377.

20. Nguyen Minh D, Pouya A. Une méthode d'étude des excavations souterraines en milieu viscoplastique. Prise en compte d'un état stationnaire des contraintes. Revue Francaise de Geotechnique 1992; 59: 5-14.

21. Giraud A, Rousset G. Time-dependent behaviour of deep clays. Engineering Geology 1996; 41(1-4):181-195.

22. Simo JC, Hughes TJR. Computational Inelasticity. Springer: Berlin, 1997.

23. Panet M. Le calcul des tunnels par la méthode convergence-confinement. Presse de l'Ecole Nationale des Ponts et Chaussées, 1995 (in French).

24. Castem-A finite element package. http://www.castem.org:8001 (10 February 2001).

25. Gill PE, Murray W, Wright MH. Practical Optimization. Academic Press: New York, 1981.

26. Scilab - a free CACSD Package by INRIA http://www-rocq.inria.fr/scilab/ (10 February 2001).

27. Hoteit N, Ozanam O, Su K, Chiarelli AS. Thermo-hydro-mechanical behaviour of deep argillaceous rocks. In Pacific Rocks 2000, Girard J, Liebman M, Breeds C, Doe T (eds). A. A. Balkema: Rotterdam, 2000; 1073-1078.

28. Bérest P, Nguyen Minh D. Time dependent behaviour of lined tunnels in soft rocks. In Proceedings of the Eurotunnel'83 Conference. Basle, 1983: 57-62.

29. Lorenzi A, Yakhno VG. An identification problem related to isotropic non-homogeneous stratified viscoelastic cylindrical body. Journal of Inventory Ill-Posed Problems 1997; 5(1):29-53.

30. Janno J, Von Wolfersdorf L. An inverse problem for identification of a time- and space-dependent memory kernel in viscoelasticity. Inverse Problems 2001, 17(1):13-24.

31. Lecampion B, Constantinescu A, Nguyen Minh D, Allagnat D. On the determination of elastoviscoplastic constants of hard marls from in situ measurements performed on a motorway tunnel in the Alps, North American Rocks Mechanics Symposium, Toronto, 2002. Proceedings, Hammah et al. (eds), ISBN 077276708 4; vol 1: 251-258. 